http://dx.doi.org/10.35381/racji.v5i8.651

\title{
Deterioro de los activos en el marco de las normas internacionales de información financiera
}

\section{Impairment of assets under international financial reporting standards}

\author{
Raysa Chiquinquirá Chirino-García \\ rchirino@urbe.edu.ve \\ Universidad Dr. Rafael Belloso Chacín, Maracaibo \\ Venezuela \\ https://orcid.org/0000-0002-0336-2870
}

Recibido: 10 de noviembre de 2019

Aprobado: 15 de diciembre de 2019

\section{RESUMEN}

Esta investigación tuvo como objetivo analizar el deterioro de la Propiedad, planta y equipos (PPE) en las empresas del ramo carpintero en el Municipio Maracaibo, estado Zulia, dado que este ramo industrial tiene la disyuntiva por la adopción y aplicación de las Normas internacionales de Información Financiera, específicamente la (NIC 36) inherente al deterioro de los activos. En consideración con los aportes de Vázquez y Díaz (2013), Mantilla (2006), Romero (2012) y Chirino Marrufo (2017), quienes aportaron teorías para este estudio y suministraron información relevante libre del encubrimiento de pérdidas financieras en unidades generadoras de efectivo; dado que, la PPE se deben evaluar para diagnosticar indicios de deterioro por factores internos y externos, así como los ajustes contables respectivos.

Descriptores: Deterioro; unidad generadora de efectivo; ramo carpintero.

\begin{abstract}
The objective of this research was to analyze the deterioration of Property, Plant and Equipment (PPE) in the companies of the carpentry branch in the Municipality of Maracaibo, Zulia State, given that this industrial branch has the dilemma due to the adoption and application of the International Standards of Financial Information, specifically the (IAS 36) inherent to the impairment of the assets. In consideration with the contributions of Vázquez and Díaz (2013), Mantilla (2006), Romero (2012) and Chirino Marrufo (2017), who contributed theories for this study and provided relevant information free of the cover-up of financial losses in generating units of cash; since, the PPE must
\end{abstract}


be evaluated to diagnose signs of deterioration by internal and external factors as well as the respective accounting adjustments.

Descriptors: Deterioration; cash generating unit; carpenter branch.

\section{INTRODUCCIÓN}

Los estados financieros de las empresas del ramo Carpintero, algunos son elaborados bajo los lineamientos establecidos en las Normas Internacionales de Información Financieras (NIIF), siendo de particular interés la Norma Internacional de Contabilidad referida al Deterioro de Activos (NIC 36), la cual establece entre otros aspectos relevantes, que las propiedades, plantas y equipos (PPE) no pueden ser registradas por montos que superen sus valores de recuperación; ello es, el mayor valor entre el valor recuperable y el valor de uso, De ahí que, surgió la necesidad de aplicar la NIC 36, cuyo objetivo es establecer los procedimientos que una entidad aplicará a las propiedades planta y quipos, así como los inventarios para satisfacerse que sus activos están contabilizados por un importe que no sea superior a su importe recuperable.

Es de hacer notar que, la complejidad para la implementación de esta norma producto de una serie de tareas, revisten la importancia estudiar el deterioro de los activos además del valor de continuación puesto que la gerencia tiene la disyuntiva de ajustar, desincorporar o desincorporar un activo así como revelar dichas situaciones en los Estados Financieros del ente.

Es perentorio que, el valor de los activos en relación con el valor neto muestre en los libros contables una seguridad razonable que evite la coexistencia de pérdidas ocultas en las cifras mostradas en los estados financieros. En este sentido, para dar confianza tanto a los accionistas como a todos los interesados en la información financiera de la entidad, ésta deberá incorporar los test que coadyuven a lograr la valoración de esos activos realizando estudios mediante técnicas que le permitan establecer la razonabilidad de las cifras. 
Dentro de este contexto, para que la información financiera proporcione confiabilidad, la gerencia deberá tener cautela ante los indicios de deterioro u obsolescencia de sus activos ya sea para ajustarlos, para hacer las revelaciones en los estados financieros de los criterios seguidos así como la baja de valor en los mismos. De ahí que, el monitoreo periódico de indicios de deterioro ejecutados en diferentes unidades de negocio e inversiones se transforma en una herramienta de control, así como de gestión en obsequio a principios de transparencia gerencial.

Es de hacer notar que, para la legislación tributaria venezolana, el deterioro de los activos, no es admitido como costo o gasto deducible para efectos de la determinación y declaración del impuesto sobre la renta; sin embargo, dado el principio de transparencia gerencial, se requiere mostrar la verdadera situación financiera de las empresas; por lo que, resultó pertinente la realización del presente estudio.

Esta investigación tomó como objetivo general, analizar el deterioro de la Propiedad, planta y equipos (PPE) en las empresas del ramo carpintero en el Municipio Maracaibo, estado Zulia, con particular interés a la Norma Internacional de Contabilidad referida al Deterioro de Activos (NIC 36), en el marco del cumplimiento de la misma para considerar el impacto contable y financiero del deterioro, así como la obsolescencia, a la luz de una visión ampliada del tema in comento. De manera que, el estudio de deterioro de los activos, permite suministrar información financiera de manera relevante y confiable para evitar el encubrimiento de pérdidas en los estados financieros.

Adicionalmente, esta investigación posibilita el conocimiento de la capacidad que tiene una empresa para la generación de efectivo; en virtud de lo cual, resulta perentorio que los activos de las empresas del ramo sean evaluadas por especialistas con el fin de obtener los diagnósticos respectivos para valorar si existen indicios de deterioro ocasionados por factores internos y externos, tales como: medio ambiental dentro de este se identifica la exposición al sol, lluvias, aguas sulfatadas, manejo inadecuado de los equipos, entre otros, además de la obsolescencia de los equipos causado por el uso intensivo u horas máquinas. 


\section{Descripción de la problemática}

Las Normas Internacionales de Información Financiera (NIIF) son preceptos contables emitidos por el Consejo de Normas Internacionales de Contabilidad (sus siglas en inglés IASB) con el propósito de estandarizar o unificar la aplicación de normas contables en el mundo. Dentro de este contexto, se puede advertir que son globalmente aceptadas, comprensibles y de alta calidad. De ahí que, las NIIF permiten que la información de los estados financieros sea comparable, así como transparente, lo que coadyuva a la toma de decisiones de inversores además de participantes en los mercados de capitales de todo el mundo.

En el mismo orden de ideas, las (NIIF), adoptadas por el Consejo Internacional de Normas de Contabilidad, comprenden: a) NIIF, b) Normas Internacionales de Contabilidad c) interpretaciones originadas por el comité de interpretaciones de Normas Internacionales de Información Financiera (CINIIF) o el anterior Comité permanente de Interpretación. De manera que estos estándares son normas exigibles, globalmente aceptadas basadas en principios claramente articulados y exigen que los Estados Financieros contengan información de indubitable calidad que facilite la toma decisiones económicas en un ente.

Para la aplicación de estas normas en Venezuela, se realizó una auscultación e interpretación exhaustiva, por parte del Comité Permanente de Principios de Contabilidad (CPPC) y aprobadas en Directorio Nacional Ampliado (DNA); identificándose con las siglas VEN-NIF a los Principios de Contabilidad Generalmente Aceptados en Venezuela, que comprenden cada una de las normas antes señaladas así como los boletines de Aplicación identificados con las siglas BA VEN-NIF, previamente aprobados en un directorio nacional ampliado.

Desde esta perspectiva, los Estados Financieros en Venezuela, deben ser elaborados bajo los lineamientos establecidos en las Normas Internacionales de Información Financieras (NIIF), siendo de particular interés para esta investigación, la Norma Internacional de Contabilidad referida al Deterioro de Activos (NIC 36) concatenada 
con la (NIC 16), ya que entre otros aspectos relevantes, se establece que las Propiedades, Plantas y Equipos (PPE) no pueden ser registradas por montos que superen sus valores de recuperación; ello es, el mayor valor entre el valor recuperable y el valor de uso.

En este sentido, las actividades de las industrias dedicadas al ramo Carpintero dadas las características de su PPE, requieren que este rubro sea monitoreado periódicamente por la gerencia con el fin de determinar de manera oportuna los indicios de deterioro y prever posibles pérdidas ocasionadas ya sea por el uso, tiempo o agotamiento de la vida útil. De ahí que, resulta perentorio aplicar la NIC 36, cuyo objetivo es establecer los procedimientos que una entidad deberá adoptar a las propiedades, planta y quipos, así como a los inventarios para satisfacerse que sus activos están contabilizados por un importe que no sea superior a su importe recuperable.

Dentro de este contexto, la adopción de ésta genera una variedad de situaciones cuyas implicaciones contables y financieras pueden aparejar impactos significativos en las cifras mostradas en los estados financieros. Es de hacer notar que, la complejidad para la implementación de esta norma producto de una diversidad de tareas, revisten la importancia estudiar el deterioro de la Propiedad, Planta y Equipo (PPE) además de considerar el valor de continuación como activo; puesto que, la gerencia se enfrentará al reto; en momentos de turbulencia económica, social y política, de ajustar, desincorporar o dar de baja a un activo así como revelar dichas situaciones en los Estados Financieros del ente.

Resulta perentorio que, el valor de la PPE en relación con el valor neto muestre en los libros contables una seguridad razonable que evite el mantener pérdidas ocultas en las cifras que conforman los Estados Financieros. En este sentido, para dar confianza tanto a los accionistas como a todos los interesados en la información financiera de la empresa, ésta deberá incorporar pruebas o test que coadyuven a lograr la valoración de esos activos realizando estudios mediante técnicas que le permitan establecer la razonabilidad de las cifras mostradas en los estados financieros. 
Desde esta perspectiva, para dar cumplimiento a las estipulaciones contempladas en las normas internacionales de información financiera, aunado al ordenamiento jurídico venezolano, para que ésta proporcione confiabilidad y fiabilidad, la gerencia deberá tener cautela ante los indicios de deterioro u obsolescencia en la Propiedad, Planta y Equipo ya sea para ajustarlos además de hacer las revelaciones en la información financiera, los criterios seguidos, así como la baja de valor en los mismos. En esta instancia, el monitoreo periódico de los indicios de deterioro ejecutados en diferentes unidades de negocio e inversiones se transforma en una herramienta de control, así como de gestión en obsequio a los principios de transparencia gerencial.

Ha llegado el momento de resaltar que, para la legislación tributaria venezolana, el deterioro de los activos, no es admitido como costo o gasto deducible para efectos de la determinación y declaración del impuesto sobre la renta. Sin embargo, dada la coexistencia del principio de transparencia organizacional, se requiere mostrar la verdadera situación financiera en las empresas, De ahí que, resulta pertinente la realización del presente estudio.

En otro orden de ideas, el deterioro viene dado por factores internos o externos, siendo los primeros: el paso del tiempo, la falta de mantenimiento a la PPE, restauración de infraestructuras, prácticas inadecuadas al momento de manipular los equipos, incendios, entre otros. Ahora bien, dentro de los externos está el factor medio ambiental, tales como: la exposición al sol, aire, lluvias, entre otros, los cuales aparejan la disminución de la vida útil de la Propiedad, Planta y Equipos, así como el inventario de repuestos y componentes.

En el mismo orden de ideas, es ineludible abordar en esta investigación el estudio de la referida norma aplicable a las industrias del ramo Carpintero en el Municipio Maracaibo del Estado Zulia, dado que en los últimos años los niveles de producción han experimentado bajas significativas; por lo que, se presume que las mismas pueden ser a causa del factor de deterioro u obsolescencia de la Propiedad, Planta y Equipos (PPE). 
En esta investigación se tomará como objetivo general, analizar el deterioro de la Propiedad, Planta y Equipo (PPE) en el marco de las Normas Internacionales de Información Financieras (NIIF), con particular interés en la Norma Internacional de Contabilidad referida al Deterioro de Activos (NIC 36) concatenada con la (NIC 16), en el marco del cumplimiento de la misma a fin de considerar el impacto contable así como financiero del deterioro y obsolescencia, a la luz de una visión ampliada del tema in comento.

Sobre la base de las ideas planteadas anteriormente y desde la perspectiva contextual de la situación problema antes explanada, se formula la interrogante de la investigación siguiente: ¿De qué manera el deterioro de la Propiedad, Planta y Equipo incide en las cifras mostradas en los Estados Financieros en las empresas del ramo carpintero en el municipio Maracaibo?

\section{Objetivo general}

Analizar el deterioro de la Propiedad, planta y equipos (PPE) en las empresas del ramo carpintero en el Municipio Maracaibo, estado Zulia.

\section{Objetivos específicos}

Analizar la Propiedad, Planta y Equipo (PPE), desde la óptica de las Normas Internacionales de Información Financiera "NIIF", en las empresas del ramo carpintero en el Municipio Maracaibo, estado Zulia.

Generar lineamientos teóricos-prácticos en el marco de la Normas Internacionales de Información Financiera aplicables a la Propiedad, Planta y Equipos en empresas del ramo carpintero en el Municipio Maracaibo, estado Zulia.

\section{Fundamentación de la investigación}

Desde la perspectiva teórica, esta investigación constituye un instrumento de valor para la comunidad científica; por cuanto brindará una fuente de consulta para otros estudios 
relacionados con el deterioro de la Propiedad, Planta y Equipo (PPE) además estará soportada por información actualizada, así como planteamientos de diversos autores especialistas en la materia, lo cual permitirá confrontar las sustentaciones teóricas de estos, traduciéndose en conocimientos importantes.

Dentro de este marco, este estudio pretende proponer lineamientos teóricos-prácticos en el marco de la Normas Internacionales de Información Financiera aplicables a la Propiedad, Planta y Equipos; por lo que representará un aporte al sector empresarial pues mediante la aplicación de éstos en el sector empresarial coadyuvarán a suministrar información financiera relevante además de confiable evitando el encubrimiento de pérdidas en los estados financieros.

Asimismo, posibilitará el conocimiento de la capacidad que tiene una organización en la generación de efectivo. Asimismo, se requerirá la participación de terceros que efectúen los diagnósticos respectivos que permitan valorar la existencia de indicios de deterioro ocasionados por factores internos y externos. Además, esta investigación constituye un aporte metodológico, por cuanto utilizará un instrumento de recolección de datos y medición que servirá para generar los lineamientos teóricos-prácticos en el marco de la Normas Internacionales de Información Financiera aplicables a la Propiedad, Planta y Equipos. Del mismo modo, servirá de guía para otras investigaciones vinculadas con la variable objeto de estudio.

Desde el punto de vista social, el presente estudio constituye una guía que ayudará a las empresas del ramo carpintero que tengan inconvenientes con el deterioro de la Propiedad, Planta y Equipo (PPE) para que esta información financiera sea medida y revelada de manera razonable evitando la ocultación de pérdidas, lo cual no representa una sana medida de control interno ni revela información fiable basa para la toma de decisiones oportunas. Adicionalmente, servirá de aporte a la comunidad científica, así como a los conocedores de su contexto y realidad, para que sean capaces de reflexionar sobre los aspectos que deben ser atendidos logrando con la adopción del principio de transparencia gerencial. 


\section{ASPECTOS METODOLÓGICOS}

Esta investigación se encuentra definida por el contenido que se aborda; por lo que, el tipo de investigación es documental. En este sentido, Arias (2012) la define como el proceso basado en la búsqueda, recuperación, análisis, crítica e interpretación de datos secundarios, ello es, los obtenidos y registrados por otros investigadores en fuentes documentales: impresas, audiovisuales o electrónicas. Del mismo modo, es un estudio descriptivo puesto que para Tamayo y Tamayo (2007), comprende la descripción, registro, análisis e interpretación de la naturaleza actual, la composición de los procesos o fenómenos, trabaja sobre las realidades de hechos y su característica fundamental es presentar una interpretación correcta.

En razón de ello, el tipo de investigación se estructura por la complementariedad, en una investigación documental bibliográfica y descriptiva utilizando la hermenéutica, donde se analizó desde una perspectiva determinista al asumir un cuadro de categorías que orientó la investigación.

\section{APROXIMACIONES TEÓRICAS}

Con el fin de efectuar el abordaje sobre el análisis de la Propiedad, Planta y Equipo (PPE), desde la óptica de las Normas Internacionales de Información Financiera "NIIF", en las empresas del ramo carpintero, resulta perentoria la conceptualización del rubro desde la óptica de las Normas Internacionales de Información Financiera "NIIF", para evitar confusiones, dado que es un término genérico reflejado en el estado de situación financiera como inversión permanente, también es considerado como bienes tangibles conocidos también en el ramo comercial o empresarial e industrial como activos fijos.

Ahora bien, desde la perspectiva teórica de las Normas Internacionales de Información Financiera "NIIF", específicamente la Norma Internacional Contable "NIC", número 16, Moreno (2011) comenta que "estos activos; ello es, los inmuebles, planta y equipos, son bienes tangibles cuyo objeto es: a) el uso o usufructo de los mismos en beneficio de la 
entidad, b) la producción de artículos para su venta o para el uso de la propia entidad, c) la prestación de servicios a la entidad, a su clientela o al público en general.

Desde otra perspectiva, la Revista Gerencie (2014), señala que la Propiedad, Planta y Equipo se corresponde con la definición clásica e incluye todos los bienes que van a ser usados en el proceso productivo normal de la empresa. Las inversiones se van a recuperar a través del ciclo de explotación de la empresa; además, se encuentra clasificada como activo no corriente o fijo. (Disponible en: www.gerencie.com/propiedadplanta-y-equipo-clasificacion.html. Consultada el 21/11/2017).

En el mismo orden de ideas, para Deloitte (2016), las propiedades, planta y equipos pueden definirse como todo bien tangible, de naturaleza relativamente permanente, los cuales son usados o se usarán en la explotación o giro comercial del negocio con el fin de generar ingresos y sobre los cuales no se tiene la intención de venderlos. (Disponible en www2.deloitte.com/hn/es/pages/audit/articles/niif-bolsillo.html. Consultada el 21/11/2017).

Volviendo la mirada a lo planteado anteriormente, se puede acotar que la adquisión de esos bienes revela que, en el curso normal de las operaciones de la entidad, su propósito es de utilización más no de venta. En este sentido, esta conceptualización puede otorgar mejoras a uno de los problemas vinculados con este rubro, inherente al reconocimiento de los activos, determinación de las cantidades cargadas, así como los cargos por depreciación además de las pérdidas por deterioro, entre otros.

Ha llegado momento de comentar que, las "NIIF" como estándar internacional, cambia la visión, así como la clasificación de los elementos de Propiedad, Planta y Equipo; ello es, atendiendo ya no a sus características, sino al destino dentro de la entidad y, más que a su empleo o uso. En otras palabras, a la forma en que van a generar los flujos de caja, como corresponde a un sistema contable eminentemente financiero. Por lo tanto, se pasa de una sola categoría dentro del estado de situación financiera, propiedad, planta y equipo, a referir con, al menos, tres posibilidades de ubicación de los mismos bienes. 
En esta instancia se fija posición con lo comentado por Moreno (2011), quien en su enunciado advierte las tres posibilidades de ubicación de los referidos bienes, tal es el caso de: a) el uso o usufructo de los mismos en beneficio de la entidad, b) la producción de artículos para su venta o para el uso de la propia entidad, c) la prestación de servicios a la entidad, a su clientela o al público en general.

Dentro de otro contexto, la NIC 16 referida a PPE, contempla como requerimiento que las empresas identifiquen y contabilicen por separado los "componentes importantes" del activo, en el caso que nos ocupa, ello se puede referenciar con el ejemplo siguiente: en el departamento de corte y armado, el equipo de corte está conformado por componentes, tales como: canteador, sierra de cinta e incluso la sierra radial y las hojas individuales. En este sentido, para la determinación de qué es "importante", resulta exigible un juicio, lo cual a su vez genera una serie de problemas prácticos, tales como la forma de abordar las necesidades de datos relacionados y los requerimientos de procesamiento del sistema; puesto que, esos componentes individuales requieren que sean depreciados por separado y castigados individualmente, dado que se reemplazan con el tiempo. Por consiguiente, una vez que se determinan los componentes, se necesitará actualizar los sistemas para reflejarlos con sus respectivas vidas depreciables.

Ahora bien, en el caso in comento del equipo de corte, es muy probable que el proveedor indique la duración aproximada según las horas diarias de uso; puesto que, cada tipo de activo tendrá una estimación diferente y resulta relevante que la empresa no generalice este aspecto; ello es, no es recomendable que se establezca una política general para todas las máquinas ya que la vida útil debe documentarse según las características del activo así como el uso que la gerencia estime darle.

Con base a lo anterior, se resalta la importancia de este aspecto puesto que la vida útil está íntimamente relacionada con el valor residual ya que son inversamente proporcionales, pues a menor vida útil, mayor valor residual. Cabe resaltar que, para efectos de depreciación, cuando se trata de activos cuya utilización es baja o son poco utilizados, las NIIF son muy claras, pues indican que la depreciación inicia cuando el 
activo está disponible para ser utilizado; ello es, cuando se encuentra listo y en condiciones esperadas y requeridas por la Gerencia; esa depreciación continúa hasta tanto el activo se desincorpore o dé de baja.

En resumen, las NIIF castigan esos activos ociosos al aplicar el criterio que si el activo no está generando beneficios debe ser excluido de los estados financieros ya que la depreciación no cesa cuando el activo está sin utilizar; puesto que, el hecho de estar ocioso no implica que se deba detener su depreciación, es por ello que la entidad debe seguir depreciándolo hasta su desincorporación.

Se añade a lo anterior, que esta fundamentación está basada en la definición aportada anteriormente ya que precisamente involucra la generación de beneficios económicos para la entidad; por lo tanto, para esa PPE ociosa hay que evaluar el valor del deterioro de esos activos; si no los está utilizando, ni los va a usar en un futuro previsible, lo mejor es venderlos y convertirlos en recursos líquidos que se puedan aprovechar para la operación.

Sin embargo, la norma en el párrafo 17.20 señala la excepción para aquellos activos que en algunos casos la depreciación puede ser en función del uso o de las unidades producidas; siendo así, no hay producción; por consiguiente, el cargo por depreciación será nulo. De ahí que, esta será la forma más acertada de depreciar aquellos bienes que no son necesarios para la empresa; por tanto, no se utilizan con frecuencia, con lo cual dado el poco uso, se frenaría el deterioro.

Ha llegado el momento de efectuar el abordaje sobre el deterioro y su impacto contablefinanciero a la luz del marco normativo establecido en las Normas Internacionales de Información Financieras (NIIF), específicamente en la Norma Internacional de Contabilidad referida al Deterioro de Activos (NIC-36), de manera que es perentorio entender algunas conceptualizaciones que se relacionan y utilizan en esta investigación. Con respecto al Deterioro de la (PPE), vale la pena desglosar este término con el propósito de lograr una mayor compresión del mismo. En este sentido, se puede acotar que el deterioro es la condición existente, cuando el valor de recuperación de los "activos 
de larga duración" en uso o en disposición son menores a su "valor neto en libros"; ello es, se impactan los beneficios económicos futuros; lo anterior concuerda con lo planteado por Romero (2012), quien sostiene que la pérdida por deterioro es el monto en que los beneficios económicos futuros esperados de unos bienes son menores que su valor neto en libros; por lo que, ésta puede deberse a obsolescencia, daños a las maquinarias o a los artículos en su valor de mercado.

Por su parte Vázquez y Díaz (2013), comentan que con el paso del tiempo los bienes cambian su valor en el mercado, que en la mayoría de los casos son a causa del deterioro, su valor disminuye, pero existen algunas excepciones como las antigüedades, obras de artes o artículos que estaban en desuso que se convierten en cosas de uso continuo a una nueva moda.

Sobre la base de las ideas planteadas anteriormente, se puede acotar que la amenaza de deterioro en un activo e incrementa en las empresas del ramo carpintero puesto que tiene un valor recuperable susceptible a los problemas inherentes a la producción, medioambientales, entre otros. De ahí que, es perentoria la intervención del juicio de un experto para poder establecer un modelo de evaluación eventual de deterioro que abarque los procedimientos establecidos en la NIC 36, los cuales deberá aplicar para cerciorarse si sus activos se han contabilizados por montos superiores al valor recuperable.

En esta instancia, para la investigadora, resulta necesario que para cada ejercicio económico, la gerencia del ramo Carpintero deberá realizar una nueva evaluación del valor neto de realización con el propósito de determinar cuándo han dejado de existir las circunstancias que dieron origen a una pérdida por deterioro de los activos aunado a lo anterior la gerencia de la entidad deberá tener prudencia ante cualquier señal que haga suponer la existencia de posibles deterioros de los activos.

En este sentido, Mantilla (2006), comenta lo señalado en la NIC 36, como condición suspensiva; en otras palabras, que si y solo si, la cantidad recuperable de un activo es menor que su cantidad cargada, habrá que reducir hasta su importe recuperable; en 
razón de ello, la investigadora considera que esa reducción es una pérdida por deterioro, la cual tiene que cargarse inmediatamente ya sea en utilidad o pérdida, a menos que el activo se cargue a su cantidad revaluada según otro Estándar. Para resumir y a manera proporcionar un diagnostico que adviertan las señales o síntomas del deterioro además de las reglas para su evaluación, en el próximo diagrama, se ilustra un extracto para una mejor visualización.

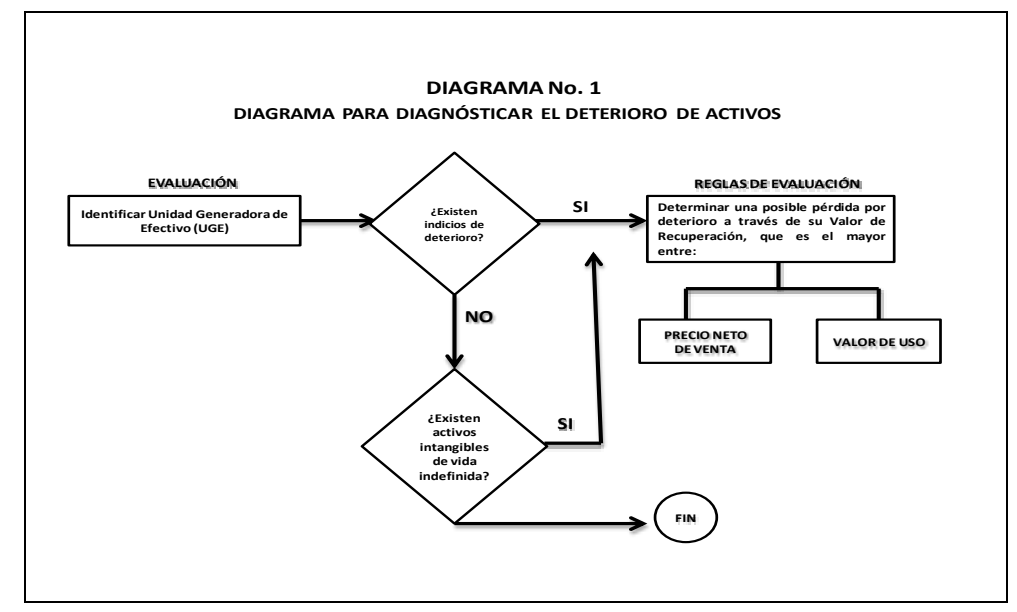

Fuente: Chirino Marrufo (2017)

Desde otra perspectiva, como sana medida gerencial, para poder enfrentar los indicios de deterioro, la administración de la empresa deberá diseñar además establecer por escrito una serie de políticas, procedimientos, guías y criterios, para definir los controles internos que coadyuven periódicamente la realización de pruebas o test de deterioro de los activos.

A lo anterior se agrega, los riesgos de incorrección material, los cuales pueden ser el resultado de estimaciones contables incorrectas originadas por una inadecuada interpretación de los hechos que la generaron o debido a criterios administrativos inherentes a la determinación de estimaciones contables no razonables o inapropiada selección y aplicación de las políticas contables de la entidad. En este sentido, el riesgo de errores significativos puede experimentar incrementos, lo cual apareja requisitos 
contables y de información financiera más complejos para el cálculo, análisis, así como la determinación del deterioro de activos.

En este sentido, Chirino Marrufo (2017) sostienen que el rol de la administración de la entidad, es relevante, pues deberán capacitar técnicamente al personal, para poder llevar a cabo el análisis correspondiente de los activos sujetos al deterioro además de monitorear la valuación y revelaciones del deterioro de activos presentadas en los estados financieros además podrán incluir revelaciones con respecto a:

a) Métodos adecuados de valuación que incluya los supuestos relacionados utilizados por la gerencia, para calcular el deterioro de activos, así como la consistencia en la aplicación de los mismos.

b) Lo pertinente de las revelaciones vinculadas con el deterioro de los activos.

c) La necesidad de informar los efectos que surjan por eventos posteriores o que aparejen algún ajuste a la valuación y/o revelaciones en los estados financieros.

Cabe resaltar que, la NIC 36 define el valor en uso de un activo, como el valor presente de los flujos futuros de efectivo estimados que se espera obtener de un activo o unidad generadora de efectivo; por lo que, es imprescindible conocer el valor de uso, para Mantilla (2006) citado por Chirino Marrufo (2017), la NIC 36 plantea que hay que considerar una serie de elementos, los cuales se tienen que reflejar en el cálculo del valor en uso de un activo, dentro de los cuales se tienen:

a. Un estimado de los flujos de efectivos futuros que la entidad espera obtener del activo.

b. Expectativa sobre las posibles variaciones en las cantidades 0 en la oportunidad de esos flujos de efectivos futuros.

c. El valor del dinero en el tiempo, representado por la tasa de interés libre de riesgo del mercado actual.

d. El precio por superar la incertidumbre inherente al activo.

e. Otros factores, tales como liquidez, que los participantes del mercado reflejarían en la fijación de los precios de los flujos de efectivo futuros que la 
entidad espera obtener del activo.

Del mismo modo, Mantilla (2006) citado por Chirino Marrufo (2017), sostiene que, la estimación del valor de uso de un activo conlleva los pasos siguientes: en primer lugar, la estimación de las entradas y salidas de efectivo futuras, las cuales se han de obtener a partir del uso continuo del activo y su última disposición; en segundo lugar, la aplicación de la tasa de descuento apropiada para esos flujos de efectivos futuros, tales estimaciones no incluyen los flujos provenientes de las actividades de financiación ni por cobro o pago de impuestos a las ganancias.

En este sentido, la norma aclara que los flujos de efectivo futuros se estimarán, para el activo, teniendo en cuenta su estado actual, de manera que estas estimaciones no incluirán entradas o salidas de efectivo futuras provenientes o que surjan de:

(a) una reestructuración futura en la que la entidad no se ha comprometido todavía; o

(b) mejoras o aumentos del rendimiento de los activos.

La investigadora sostiene que para poder llevar a cabo la evaluación de los indicios del deterioro, se deberán considerar algunos aspectos claves, los cuales se muestran en el tes para conocer los indicios de deterioro, identificado como Anexo No. 1, el cual contempla ¿Cuentan con la Materia prima suficiente para elaborar sus productos y así cubrir los costos operativos, generando rentabilidad a la organización?, ¿Se realizan los mantenimientos preventivos a la unidad generadora de efectivo?

A lo anterior se agrega que, se debe conocer si, ¿Se efectúan las estimaciones de los flujos de efectivos futuros que la entidad espera obtener de los activos?, ¿Analizan las expectativas sobre las posibles variaciones en las cantidades o en la oportunidad de esos flujos de efectivos futuros?, ¿Evalúan el valor del dinero en el tiempo, representado por la tasa de interés libre de riesgo del mercado actual?, ¿Cuáles son las presunciones relevantes de la administración para determinar el deterioro de los activos?; ¿Qué técnicas utiliza la gerencia para monitorear los cambios o indicios de deterioro?; ¿Qué 
resultados espera obtener una vez culminado el tes o prueba de los activos expuestos al deterioro?.

Ha llegado el momento de definir la unidad generadora de efectivo, de acuerdo con la NIC 36 que la concibe como el grupo identificable más pequeño de activos que genera entradas de efectivo, los cuales son en buena medida independientes de las entradas de efectivo provenientes de otros activos o grupos de activos.

En esta instancia, la investigadora infiere que al momento de realizar dichas proyecciones se podrá conocer la capacidad generadora de efectivo del ramo carpintero, a fin de identificar si hay indicios o deterioro de las maquinarias, así como también de los equipos, las infraestructuras y el inventario de componentes, para una mejor ilustración, en el próximo cuadro, se muestran las proyecciones económicas y financieras de una unidad generadora de efectivo (UGE).

CUADRO No. 1

Determinación del Deterioro de Valor para la Unidad Generadora de Efectivo (UGE)

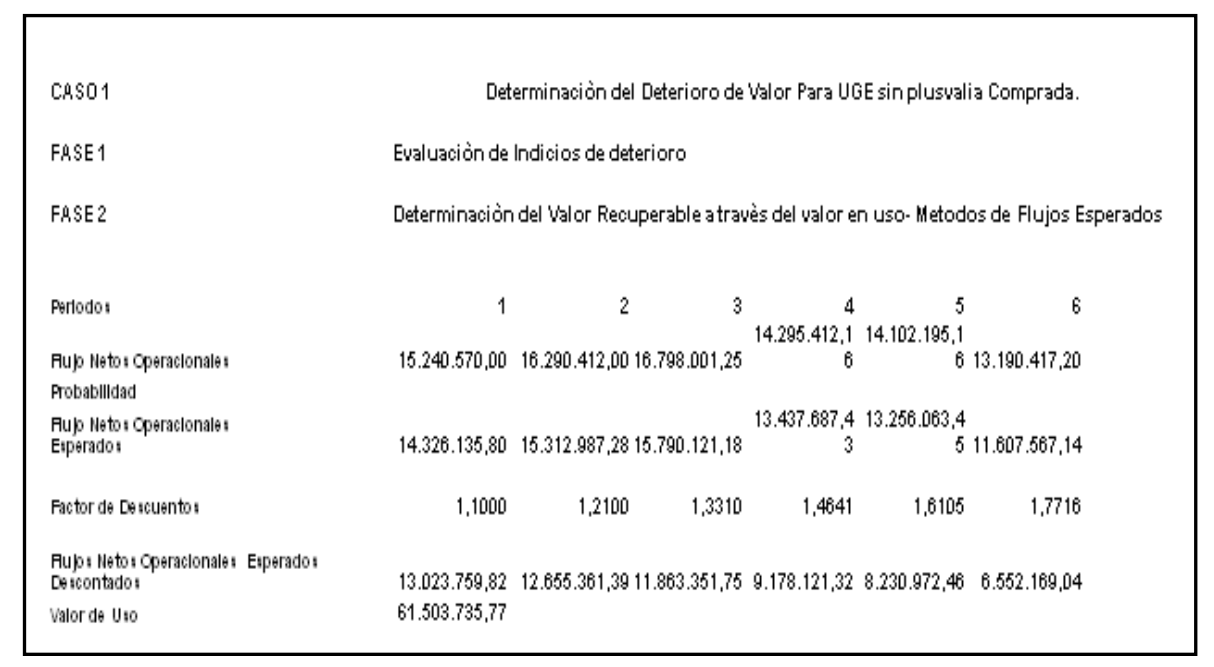

Fuente: Chirino (2018)

Sobre la base de las proyecciones anteriores, se podrá determinar la configuración de la UGE, luego la composición de la propiedad planta y equipos (PPE) con sus respectivos saldos contables, tal como se puede visualizar a continuación: 


\section{CUADRO No. 2}

Composición de la Unidad Generadora de Efectivo (UGE)

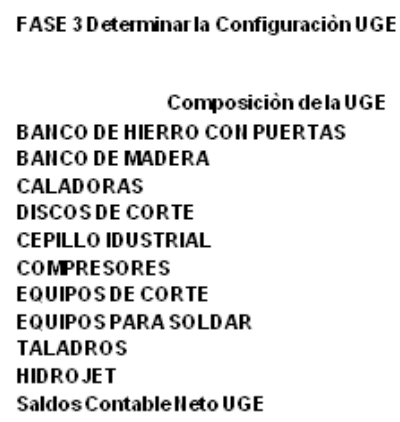

Fuente: Chirino (2018)

Una vez obtenida la composición de la UGE, nos trasladamos a la segunda fase; ello es, aplicar el test de deterioro, el cual debe llevarse a cabo cuando la empresa identifique un indicio de pérdida de valor en uno de sus activos y en caso del fondo de comercio, como mínimo debe efectuarse anualmente, este procedimiento efectuado por expertos independientes, puesto que ello aporta mayor confianza a los terceros interesados y a la alta gerencia así como a otros agentes externos, tales como: organismos reguladores, inversores, entre otros.

En consecuencia, se debe efectuar una confrontación entre el importe recuperable del activo y su valor neto contable, con el propósito de determinar sí valor neto contable del activo es superior a su importe recuperable en cuyo caso la empresa deberá deteriorar el valor en libros hasta el importe recuperable y, como máximo, hasta que el valor en libros del activo sea cero. Con el fin de brindar una mejor ilustración el próximo cuadro, muestra lo narrado anteriormente. 
CUADRO No. 3

Test de Deterioro

\begin{tabular}{|c|c|c|}
\hline \multicolumn{3}{|l|}{ Aplizar el Tes de Detericro } \\
\hline 'ilor Recuperable & $61.503725, \pi$ & \\
\hline \multicolumn{3}{|l|}{ [Strosos] } \\
\hline Sado Cortable Neto UGE & $8.578,100,100$ & \\
\hline Deteriono Del Velor & 34172944,20 & *rikterialitat \\
\hline
\end{tabular}

Fuente: Chirino (2018)

De acuerdo con las ideas planteadas anteriormente, al analizar la determinación del deterioro se evidencia en el cuadro No. 1, el valor de uso cuyas proyecciones revelan un monto de 61.503.735,77, pero el saldo contable es de $95.676 .000,00$, lo cual revela un deterioro de $34.172 .264,23$ de las maquinarias y equipos con un $36 \%$ de materialidad. De ahí que, se deberá ajustar el valor contable de la propiedad planta y equipo, tal como se muestra en el cuadro siguiente:

CUADRO No. 4

Distribución del Deterioro Valor entre los componentes de la unidad generadora de efectivo

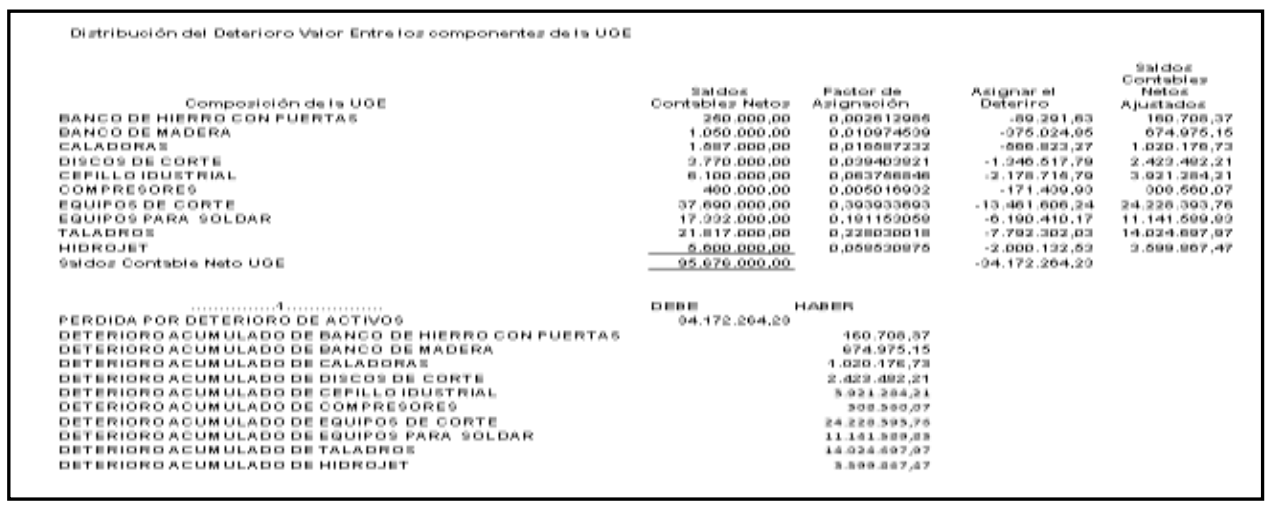

Fuente: Chirino (2018)

En síntesis, como se puede observar, una vez determinado el deterioro y ajustada la propiedad planta y equipos (PPE), esto permitirá conocer la verdadera situación financiera, así como operativa de la unidad generadora de efectivo (UGE), para poder tomar algunas decisiones en cuando a la sustitución de esos activos con el fin de mejorar 
los niveles de producción mediante la utilización de nuevas tecnologías, lo cual redunda en la disminución de los costos.

Del mismo modo, se pueden advertir otras señales que pudieran generar el deterioro de los activos, tal es el caso de la obsolescencia de la propiedad planta y equipo (PPE), Moreno (2011) citado por Chirino Marrufo (2017), comenta que cuando se decida desincorporar un activo obsoleto se debe cancelar en los libros y registros de contabilidad la inversión así como la depreciación acumulada pues el activo se debe transferir a una cuenta especial, la cual se puede denominar como "Activo fijo fuera de uso".

En esta instancia, la valuación del activo obsoleto se hará al valor neto de realización; puesto que, se reconocerá, dependiendo de las circunstancias, una pérdida o utilidad en los resultados del período. Asimismo, Ortega (2009), señala que la obsolescencia es el atraso tecnológico que van sufriendo los equipos por evolución constante de las tecnologías que se pueden adquirir en el mercado. De manera que, ese atraso tecnológico coadyuva a una peor fabricación ya sea de calidad o de diseño o en cantidad, aumento de los costos de la misma, o una disminución en los ingresos por ventas.

Por otra parte, Hamilton y Pezo (2005), advierten que son los atrasos tecnológicos por obsolescencia, la introducción de mejoras o innovaciones de los procesos productivos. Al respecto, se puede acotar que los avances tecnológicos y la apertura de los mercados ocasionan un deterioro acelerado de los activos por causas no previstas. Por otra parte, el uso de tecnologías atrasadas apareja costos elevados o ineficiencia a lo que se añaden elementos importantes, tal como el nivel de precios ya que éste incide al momento de realizar cualquier mantenimiento o reposición de partes que pudieran alargar la vida útil del activo.

En virtud de lo planteado por los autores anteriores, la investigadora fija posición con lo planteado por Hamilton y Pezo (2005), ya que resalta que el uso de tecnologías vetusta origina altos costos además de eficiencia limitada en los procesos productivos. Aunado a lo anterior, existe otro factor que apareja la obsolescencia, como es el inconveniente que se produce al momento de obtener repuestos, dada la antigüedad de esos activos. En 
este sentido, hay que considerar el entorno y la situación país; ello es, en Venezuela se presenta la dificultad para la adquisión de repuestos y partes debido a la existencia de un sistema de control cambiario, limitaciones en las empresas para ofrecer reposición de partes, componentes y repuestos, todo ello limita no solo la libertad de compra en moneda extranjera sino el mantenimiento de la maquinaria y equipos.

El problema está en que, esta situación conmina la operatividad y funcionamiento de los equipos, los cuales en su mayoría son de marcas cuyos componentes o accesorios no están disponibles en el mercado interno y para poder mantenerlos o repararlos se tiene que acudir a importación con el agravante que apareja la obtención de divisas para evitar la inoperatividad de esos activos cuyas consecuencias económicas inciden en los estados financieros. No contar con equipos aptos, aumenta los costos de mantenimiento adicionando costos de oportunidad al no contar con los equipos operativos para la generación de renta además de representar una amenaza para la pervivencia o continuidad de la entidad en el mercado.

\section{Lineamientos teóricos-prácticos en el marco de la normas internacionales de información financiera aplicables a la propiedad, planta y equipos}

En esta instancia, resulta perentorio esbozar algunos lineamientos teóricos-prácticos en el marco de la Normas Internacionales de Información Financiera aplicables a la Propiedad, Planta y Equipos (PPE) así como al deterioro de los activos en empresas del ramo carpintero, como aporte a la problemática in comento. En este sentido, la política contable deberá estar de acuerdo con lo preceptuado en las secciones 17-Propiedades, planta y equipo, 27- Deterioro del valor de los activos, 2 -Conceptos y principios fundamentales además de la sección 4 -Estado de situación financiera, y 25 Costos por préstamos de la NIIF para las Pymes. 


\section{Políticas contables}

Con respecto a las políticas contables, los elementos de la partida propiedades, planta y equipo se registran inicialmente al costo. $\mathrm{Y}$ de acuerdo con la NIIF para Pymes párrafo 17.10, en el costo se incluye el precio de adquisición que está compuesto por los honorarios legales y de intermediación, los aranceles de importación y los impuestos no recuperables, después de deducir los descuentos comerciales y las rebajas.

Por otra parte, los costos de mantenimiento menores, así como la conservación y reparación de los activos, se registran directamente en los resultados del ejercicio en el momento en que se incurren. De ahí que, para aquellos eventos ocurridos con posterioridad a su reconocimiento inicial como un activo, todas las partidas de PPE, deben ser mantenidas en libros a su costo excepto la depreciación acumulada y las pérdidas por deterioro.

Dentro de otro contexto, para los casos de renovaciones y mejoras significativas que se incurren con posterioridad a la adquisición del activo, éstas son incluidas en el valor en libros del activo o reconocidas como un activo separado, cuando aumenta la productividad, capacidad o eficiencia o se amplía la vida útil del bien. De mismo modo, es probable que generen un beneficio económico futuro, y la erogación del costo pueda determinarse de forma fiable.

\section{Reconocimiento y medición}

Con respecto al reconocimiento y medición, en las empresas del ramo Carpintero, los elementos de la propiedad, planta y equipo se reconocerán al costo como un activo si, y solo si:

a) Es probable que la Entidad obtenga los beneficios económicos futuros derivados del mismo; por lo tanto, solo se podrán reconocer en esta categoría, aquellos bienes tangibles que la empresa controle en periodos superiores a un año.

b) El costo del elemento puede medirse con fiabilidad. 
En cuanto a la Medición inicial, se medirá un elemento de propiedades, planta y equipo por su costo. Ahora bien, el costo de los elementos o componentes de la propiedad, planta y equipo comprende todo lo siguiente:

(a) El precio de adquisición, que incluye los honorarios legales y de intermediación, los aranceles de importación y los impuestos no recuperables, después de deducir los descuentos comerciales y las rebajas.

(b) Todos los costos directamente atribuibles a la ubicación del activo en el lugar y en las condiciones necesarias para que pueda operar de la forma prevista por la Gerencia. Estos pueden incluir los costos de preparación del sitio donde se ubicará el activo, los costos de entrega y manipulación inicial, los de instalación y ubicación del activo, los costos de entrega y manipulación inicial, los de instalación y montaje, los de comprobación de que el activo funciona adecuadamente.

(c) La estimación inicial de los costos de desmantelamiento o retiro del elemento, así como la rehabilitación del lugar sobre el que se asienta, la obligación en que incurre una entidad cuando adquiere el elemento o como consecuencia de haber utilizado dicho elemento durante un determinado periodo, con propósitos distintos al de producción de inventarios durante tal período.

Para la medición del costo de un elemento de propiedad, planta y equipo será el precio equivalente en efectivo en la fecha de reconocimiento. Si el pago se aplaza más allá de los términos normales de crédito, el costo será el valor presente de todos los pagos futuros. Por otra parte, para la medición posterior, la empresa medirá todos los elementos de la propiedad, planta y equipo tras su reconocimiento inicial al costo menos la depreciación acumulada así como cualesquier pérdida por deterioro del valor acumulado. En este sentido, la empresa reconocerá los costos del mantenimiento diario de un elemento de maquinaria de la propiedad, planta y equipo de las empresas del ramo carpintero, en los resultados del periodo en el que incurra en dichos costos Medición del importe recuperable: 
- El importe recuperable de un activo o de una unidad generadora de efectivo, es el mayor entre su valor razonable menos los costos de venta y su valor en uso. Si no fuera posible estimar el importe recuperable de un activo individual, las referencias contenidas en los párrafos 27.12 a 27.20 con relación a un activo también deben ser aplicables a la unidad generadora de efectivo del activo.

- De manera que el valor razonable menos los costos de ventas, es el importe que se puede obtener por la realización de un activo, en una transacción realizada en condiciones de independencia mutua entre partes interesadas y debidamente informadas, menos los costos de disposición (venta).

- Valor en uso, es el valor presente de los flujos futuros de efectivo que se espera obtener de un activo. El cálculo del valor presente involucra las siguientes fases:

(a) estimar las entradas y salidas futuras de efectivo derivadas de la utilización continuada del activo y de su disposición final; y

(b) aplicar la tasa de descuento adecuada a estos flujos de efectivo futuros.

\section{Depreciación}

En este segmento se plantea que, los activos se depreciarán a lo largo de su vida útil como activos individuales. En este sentido, si los principales componentes de un elemento de propiedad, planta y equipo tienen patrones significativamente diferentes de consumo de beneficios económicos, se distribuirá el costo inicial del activo entre sus componentes principales y se depreciará cada uno de estos componentes por separado a lo largo de su vida útil. De manera que, el cargo por depreciación se reconoce en los resultados, a menos que otra Sección de la NIIF para las Pymes permita que se capitalice como parte del costo de un activo.

De ahí que, el importe depreciable y periodo de depreciación, se distribuirá de forma sistemática a lo largo de su vida útil. El valor residual, el método de depreciación o la vida útil pueden modificarse cuando haya cambios en el uso de un activo, un desgaste 
significativo inesperado, avances tecnológicos y cambios en los precios de mercado desde la fecha sobre la que se informa más reciente.

Con respecto a los cambios in comento, se tratarán como una estimación contable (véase párrafos 10.15 a 10.18 de la NIIF para Pymes); por lo que, la depreciación de un activo comenzará cuando esté disponible para su uso y la misma cesa cuando se dé de baja o desincorpore en las cuentas. Cabe resaltar que, la depreciación no cesará cuando el activo esté sin utilizar o se haya retirado del uso, a menos que se encuentre totalmente depreciado. Así pues, para determinar la vida útil de un activo, se deberán considerar todos los factores contemplados en el párrafo 17.21 de la NIIF para Pymes, a saber:

(a) La utilización prevista del activo. El uso se evalúa por referencia a la capacidad o al producto físico que se espere del mismo.

(b) El desgaste físico esperado, que dependerá de factores operativos tales como el número de turnos de trabajo en los que se utilizará el activo, el programa de reparaciones y mantenimiento, y el grado de cuidado y conservación mientras el activo no está siendo utilizado.

(c) La obsolescencia técnica o comercial, o de los cambios en la demanda del mercado de los productos o servicios que se obtienen con el activo.

(d) Los límites legales o restricciones similares sobre el uso del activo, tales como las fechas de caducidad de los contratos de arrendamiento relacionados.

\section{Deterioro de los activos}

El tema del deterioro de los activos, es relevante y en cada fecha sobre la que se informa, se aplicará la Sección 27 -Deterioro del Valor de los Activos (de la NIIF para Pymes) con el fin de determinar si un elemento o grupo de elementos de propiedad, planta y equipo ha visto deteriorado su valor y, en tal caso, cómo reconocer y medir la pérdida por deterioro de valor.

Esta sección explica cuándo y cómo una entidad revisará el importe en libros de sus activos, cómo determinará el importe recuperable de un activo, y cuándo reconocerá o 
revertirá una pérdida por deterioro en valor. En otras palabras, cuando hayan desaparecido las causas que originaron el deterioro de valor, este deterioro se revertirá como mayor valor del activo y se abonará en la cuenta de resultados.

\section{Revelaciones}

Finalmente, las empresas del ramo carpintero revelarán al cierre de cada año, para cada categoría de los elementos o componentes de la propiedad, planta y equipo, que se considere apropiada de acuerdo con el párrafo 4.11(a), la información siguiente:

(a) Las bases de medición utilizadas para determinar el importe en libros bruto.

(b) Los métodos de depreciación utilizados.

(c) Las vidas útiles o las tasas de depreciación utilizadas.

(d) El importe bruto en libros y la depreciación acumulada (agregada con pérdidas por deterioro del valor acumuladas), al principio y final del periodo sobre el que se informa.

(e) Una conciliación entre los importes en libros al principio y al final del periodo sobre el que se informa, que muestre por separado:

(i) Las adiciones realizadas.

(ii) Las disposiciones.

(iii) Las adquisiciones mediante combinaciones de negocios.

(iv) Las transferencias a propiedades de inversión, si una medición fiable del valor razonable pasa a estar disponible (véase el párrafo 16.8 de la NIIF para Pymes).

(v) Las pérdidas por deterioro del valor reconocidas o revertidas en el resultado de acuerdo con la Sección 27-Deterioro del valor de los activos de conformidad con la NIIF para Pymes.

(vi) La depreciación.

(vii) Otros cambios. No es necesario presentar esta conciliación para periodos anteriores. 
Por otra parte, la entidad también revelará:

(a) La existencia e importes en libros de las propiedades, planta y equipo a cuya titularidad la Entidad tiene alguna restricción o que está pignorada como garantía de deudas

(b) El importe de los compromisos contractuales para la adquisición de propiedades, planta y equipo.

\section{REFLEXIONES FINALES}

En resumen, actualmente el ramo carpintero se enfrenta disyuntiva en cuanto a la contabilización y presentación del valor de sus activos, particularmente lo inherente a la Propiedad, Planta y Equipo (PPE) producto de la adopción de las normas internacionales de información financiera (NIIF) pues la norma referente al deterioro de los activos NIC 36, se concatena con la NIC 36. Adicionalmente, la aplicación de ésta genera una congerie de situaciones cuyas implicaciones contables y financieras pueden aparejar impactos relevantes en las cifras mostradas en los estados financieros.

Dado que la gerencia tendrá la alternativa de si se produce el deterioro debido a factores internos o externos tendrá que decidir si desincorpora el activo con las consecuencias derivadas de esa acción o si por el contrario le concede continuidad al mismo ya que irremediablemente a la par de ello tendrá que considerar también los altos costos que incurrirá por mantenimiento en un mercado con limitaciones cambiarias que para poder efectuar los mismos de manera preventiva o reparaciones mayores tendrá que encarar grandes obstáculos que la conducirán a castigar los resultados del ejercicio económico. De acuerdo con las ideas planteadas, la gerencia de las empresas del ramo carpintero tienen la responsabilidad de capacitar técnicamente al personal, para que puedan efectuar el análisis correspondiente de los activos sujetos al deterioro además de monitorear periódicamente, así como la realización oportuna de las pruebas o test para determinar los ajustes contables con respecto al deterioro de activos y diferenciarlos del resto de los rubros de los estados financieros. 
De manera que, para el logro de tal fin se amparará en el trabajo de expertos quienes le ofrecerán confiabilidad además le permitirá validar la razonabilidad de las cifras mostradas, los supuestos para la determinación del deterioro de activos además de la información que deberá revelar toda vez que se efectúen bajas de activos o ajustes tomando en cuenta las políticas, así como los procedimientos seguidos por el ente.

\section{REFERENCIAS CONSULTADAS}

1. Arias (2012). El proyecto de Investigación: Introducción a la investigación científica. 6ta edición. Editorial Episteme.

2. Boletín de aplicación VEN-NIF №. 0 (BA VEN-NIF 0) "Marco de Adopción de Normas Internacionales de Información Financiera.

3. Chirino y Marrufo (2017). Deterioro de los activos. Valor de continuación. Dilema Gerencial en las industrias del sector Carbonífero. Revista Internacional LEGIS de Contabilidad \& Auditoria. Publicación Trimestral Abril-Junio del 2017. Bogotá, Colombia. (ISSN 1692 - 2913).

4. Deloitte (2016). Guía rápida de NIIF. www2.deloitte.com/hn/es/pages/audit/articles/niifbolsillo.html. Consultada 21/11/2017)

5. Hamilton y Pezo (2005). Formulación y evaluación de proyectos tecnológicos empresariales aplicados. Edición Andrés Bello Colombia.

6. Internacional Accounting Standards Committee Foundation (IASCF).

7. International Accounting Standards Board. (2009). Normas Internacionales de Información Financiera aprobadas a 1 de enero de 2009. Londres: Ediciones.

8. Mantilla (2006). Estándares/Normas Internacionales de Información Financiera IFRS (NIIF). Ecoediciones. Bogotá-Colombia.

9. Moreno (2011). Contabilidad Intermedia II. Instituto Mexicano de Contadores Públicos. Grupo editorial Patria. México.

10. NIC (2004), Norma Internacional de Contabilidad № 36.

11. Normas de Presentación. Eco Ediciones. Colombia. 
12. Ortega (2009). Introducción a la contabilidad de gestión. Madrid Editorial Coslada.

13. Revista Gerencie (2014). (Disponible en: www.gerencie.com/propiedad-planta-yequipo-clasificacion.html. Consultada el 21/11/2017).

14. Romero (2012). Manual financiero para empresas. Con técnicas financieras de las mejores escuelas de negocios de Latinoamérica. Holguín Ediciones, S.A. Segunda edición.

15. Tamayo y Tamayo (2007). El proceso de la investigación científica. 4ta edición. Editorial Limusa. México.

16. Vázquez y Díaz (2013). Normas internacionales de información financiera (NIIFIFRS): Principios y ejercicio avanzados de contabilidad global.

17. Villalba (2008). Análisis de la información financiera sobre el deterioro del valor de los activos basados en la NIC-NIIF 36. Retrieved from http://bibadm.ucla.edu.ve/.

18.Zapata, (2011). Contabilidad general con base en Normas Internacionales de Información Financiera "NIIF" (7.a ed.). Editorial McGraw-Hill-Interamericana Editores. Colombia. 